SAND92-0229

Distribution

Unlimited Release

Category UC-501

Printed August 1993

\title{
THE MINIMUM DETECTION LIMITS OF RDX AND TNT DEPOSITED ON VARIOUS SURFACES AS DETERMINED BY ION MOBILITY SPECTROSCOPY
}

\author{
Phil Rodacy \\ Explosive Subsystems \& Materials \\ Sandia National Laboratories \\ Albuquerque, NM 87185
}

\begin{abstract}
An Ion Mobility Spectrometer (IMS) was used to determine the detection limits of RDX and TNT on six different substrates. The preparation of the explosive deposits on the surfaces is examined as well as effects due to the size, uniformity, method of application, and time that a deposit has been on a surface. Sampling methods are discussed along with effects of the surface topology. The transfer of explosives from a hand to a surface, and methods to reduce the detection limits are presented.
\end{abstract}




\section{DISCLAIMER}

Portions of this document may be illegible in electronic image products. Images are produced from the best available original document. 


\section{CONTENTS}

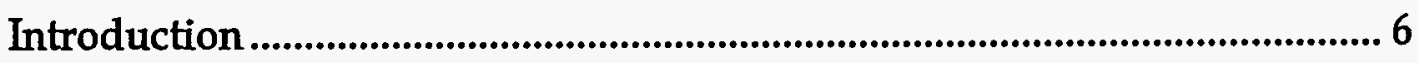

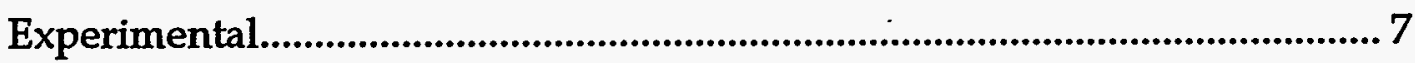

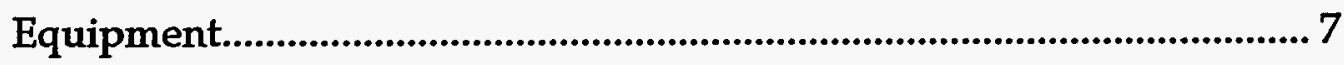

Preparation of Standards ....................................................................... 7

Preparation of Deposits ............................................................................. 8

Sampling Method .............................................................................................. 8

Substrates Examined ......................................................................................... 9

Results and Discussion ...................................................................................... 10

Detection LImit of TNT................................................................................. 11

Detection Limit of RDX.............................................................................. 12

Concentration Dependence .......................................................................... 14

Uniformity of Deposition .............................................................................. 14

Detection Limit as a Function of Time ...................................................... 15

Effect of Suface Topology ......................................................................... 15

Methods to Reduct the Detection Limit ........................................................ 16

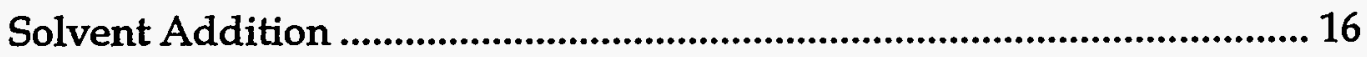

Touch Sampling ............................................................................................... 16

Transfer of Explosives by Human Touch .......................................................... 17

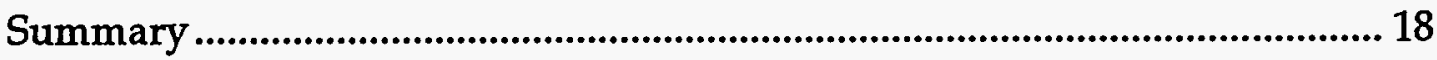

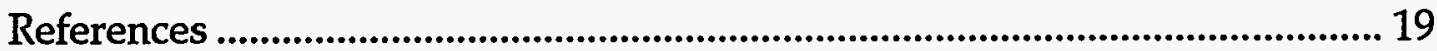

\section{FIGURES}

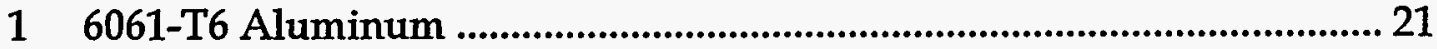

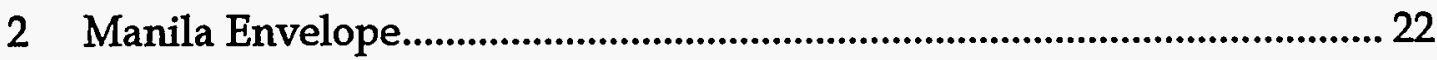

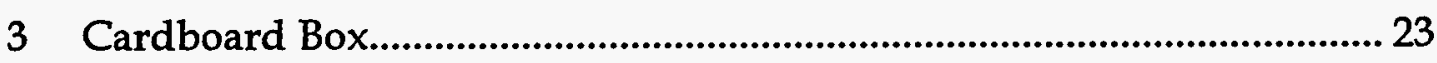

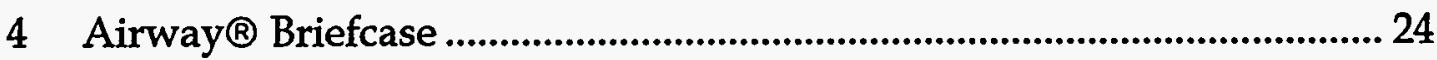

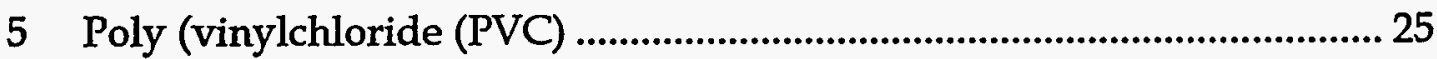

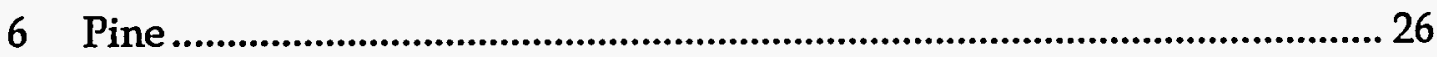

TABLES

1 Detection limits of TNT and RDX on various surfaces. .......................... 11 


\section{THE MINIMUM DETECTION LIMITS OF RDX AND TNT DEPOSITED ON VARIOUS SURFACES AS DETERMINED BY ION MOBILITY SPECTROSCOPY}

\section{INTRODUCTION}

Vapor phase sampling of explosives is of interest as a method to screen personnel, packages, vehicles, and other items for contraband explosives. Explosive molecules are expected to diffuse through containers or packages and provide a detectable amount of explosive vapor. In addition, it is likely that a person who prepares, packages, or carries explosives will inadvertently contaminate either himself and/or the exterior of the package with the explosive, thus providing a source of explosive vapor near the surface of the object. As air is collected near these contaminated items, the volatile explosive molecules can be collected, concentrated, and analyzed.

Several problems, however, can prevent the effective use of this method. It is well documented that explosive molecules are "sticky" and can adhere to various surfaces with relatively high binding energies [1], thereby precluding their detection. In addition, many explosives have an extremely low vapor pressure, which severely reduces the number of molecules available for collection and analysis. One crucial aspect of vapor phase sampling techniques is that there must be a sufficient quantity of explosive molecules present in the gas phase to be collected and analyzed. Therefore, to perform a useful screening, the analyst must have an understanding of the amount of explosive that must be present on a surface in order for it to be detected.

A study to determine the minimum detection limits of RDX and TNT deposited on various substrates has been performed. The substrates were chosen to represent a metal shipping container, a mail bomb, a boxed (cardboard) item, a wooden shipping crate, a typical briefcase, and consumer items such as radios and toys. 


\section{EXPERIMENTAL}

\section{Equipment}

This work was performed using a PCP PHEMTO-CHEM 100 Ion Mobility Spectrometer (PCP, Inc., West Palm Beach, FL) that had been modified to allow control of the reactant ion chemistry. Control of the reactant ion chemistry has been shown to be important in the analysis of explosives [2].

The IMS output signal was collected by both an IBM compatible personal computer equipped with a Phemto-Chem ASPB-1 Signal Processing Interface board and software, and a boxcar/integrator system, and processed as described in reference 3 . The sampling method used for the work described here has also been documented [3].

\section{Preparation of Standards}

Determining the minimum amount of explosive that must be present on a surface before it can be detected requires accurate deposition of a known amount of explosive on that surface. Preparation of standards for this purpose requires particular care as it has been shown that glass surfaces tend to adsorb explosive molecules [4]. The surface of the glassware, however, can be passivated and the adsorption of the explosive minimized or eliminated by either silanizing the surface with dimethyldichlorosilane or by rinsing the glassware with a concentrated solution of the explosive prior to use [4]. Essentially, these passivation procedures saturate the active sites present, thus minimizing adsorption of the explosive molecules. The effectiveness of both methods was found to be similar, so for convenience, I chose to rinse the surfaces with a concentrated solution of explosive. The details of the rinsing procedure and its effectiveness are outlined in detail in other reports [4.]. For the reader's convenience, the rinsing procedure will be summarized. 
To passivate glassware prior to preparing the standards, a concentrated solution containing approximately $1 \times 10^{-4}$ grams of explosive per microliter $(\mu \mathrm{L})$ of acetone was prepared. A TNT solution was used to prepare glassware to be used with TNT; a RDX solution was used to prepare RDX glassware. This stock solution was poured into the glassware (typically a screw-cap culture tube or small volumetric flask) and allowed to remain for approximately 10 minutes. The stock solution was then removed, and the glassware was rinsed with acetone to remove excess explosive solution adhering to the walls. The glassware was then re-filled with acetone and allowed to soak for 10 minutes. The rinsing, re-filling, and soaking with acetone was repeated for a total of three times prior to using the glassware. Previous work has shown that this procedure will passivate the glass surface, but residual explosive will not leach off the glass and into solution [4]. Pipettes and other glassware used for transferring solutions were treated in a similar manner. The glassware was allowed to dry at room temperature before use.

A standard solution with a concentration of approximately $1 \times 10^{-6}$ grams of explosive per microliter of acetone was prepared in the pre-treated glassware. Ten milliliters of acetone was then placed into a culture tube using a class A pipette, and an appropriate aliquot of the concentrated solution was added to prepare a dilute solution of the desired concentration. This dilute solution was then discarded, and a second solution of the same concentration was prepared in the same culture tube. The culture tube was not rinsed or dried after the first solution was discarded. This second dilute solution was used as a standard and deposited on the substrate, or, if necessary, diluted further using the same procedure to prepare other more dilute standard solutions. The pipettes used to transfer the solutions were pretreated as described above, and then rinsed three times with the solution being transferred. The solutions deposited on the substrates ranged between $1 \times 10^{-6}$ and $1 \times 10^{-13}$ grams of explosive per microliter of solvent.

\section{Preparation of Deposits}

To prepare explosive deposits on the surfaces for sampling, 0.02 milliliters to 0.20 milliliters of a standard solution was deposited onto the various surfaces, and the solvent allowed to evaporate as the solution was being applied to insure that the geometric area covered by 0.20 milliliters of solution was approximately the same as 
that covered by 0.02 milliliters of solution. Thus, the concentration per square millimeter could be varied while (unknown) surface effects could be minimized or at least held constant. After the solution was applied, the area that the solution covered was marked using a standard lead pencil, the area was measured, and the concentration per square millimeter calculated.

\section{Sampling Method}

After depositing the explosive solution, the substrate being tested was placed on a temperature.programmable hot plate and allowed to come to thermal equilibrium at either $25^{\circ} \mathrm{C}$ or $50^{\circ} \mathrm{C}$. The time required to attain equilibration was determined by monitoring the surface temperature of the material using a digital surface thermometer. One minute after surface equilibrium was attained, a vapor sample was collected using 6.0 inch long by 0.25 inch O.D. quartz tubes drawing air at the rate of $210 \pm 10 \mathrm{cc}$ per minute. This procedure is documented elsewhere [3]. The sampling time was 90 seconds, with the end of the quartz tube held $0.125^{\prime \prime}$ to $0.250^{\prime \prime}$ above the surface of the material being tested. Testing has shown that, when used in this manner, the quartz tubes retain between $98 \%$ (TNT) and $100 \%$ (RDX) of the explosive entering the mouth of the tube. Desorption efficiency has also been tested for the conditions employed here and is $100 \%$ for both TNT and RDX.

The quartz tube was moved in a random pattern above the explosive deposit so that all areas of the deposit were sampled numerous times during the sampling period. To approximate a field collection scenario, the samples were collected in the open laboratory. Shields or other barriers to eliminate stray air currents were not used. After collecting the sample, the explosive was desorbed from the quartz tube using the furnace system described elsewhere [3], and the data was collected using both the IMS ASPB-1 software and a boxcar integrator connected to a Spectra Physics ChromJet integrator.

\section{Substrates Examined}

The detection limits for TNT and RDX were determined for six different surfaces. These surfaces were selected to represent metal or plastic shipping containers, 
briefcases, mail and package bombs, shipping crates, and other items as listed below. The substrates, with the exception of the aluminum, were not cleaned or treated in any way. The aluminum was cleaned with acetone to remove processing oils and marking paints, and allowed to dry at room temperature before deposition of the explosive.

- A 0.020 inch thick 6061-T6 aluminum sheet was used to represent a metal shipping container, aluminum briefcase, or photography case.

- A manila envelope, such as those commonly used to mail photos, literature, correspondence, and other paper items, was selected to represent a typical mail bomb.

- A corrugated cardboard box was used to simulate a package that might contain a bomb.

- Plastic removed from the exterior of an Airway® attaché case was used to represent a typical briefcase. The plastic used for this briefcase is polyester with a carbonate filler and 1,2-benzenedicarboxylic acid dioctyl ester (dioctyl phthalate) plasticizer.

- Poly(vinylchloride) (PVC) was chosen to represent common objects such as radios, packing boxes, toys, and numerous other consumer items. Although consumer items often contain various plasticizers, modifiers, or additives, none were present in the material used for these tests.

- A wooden shipping crate was simulated by a pine veneer 0.025 inches thick. Veneer was selected so that the desired surface temperature could be reached. Thicker sections of wood acted as insulators and prevented the surface from reaching the desired temperature.

\section{RESULTS AND DISCUSSION}

The minimum detection limit was defined as being the point where a given amount of explosive deposited on a surface could be detected $50 \%$ of the time. To determine 
the detection limit, a solution was deposited on the substrate being tested, and a vapor sample taken. If a response was obtained, a less concentrated solution was used to prepare a new deposit such that the concentration per square millimeter was less, and the sampling repeated. This process was continued until the detection limit was determined. Once the detection limit was established, a minimum of eight samples were collected at this level for each explosive on each substrate. The values obtained during these tests were verified by preparing a second standard solution and repeating the analysis two to four weeks after the first value had been obtained. The average value of the responses obtained from these samples is reported as being the detection limit.

Table 1 Detection limits of TNT and RDX on various surfaces. Surface temperature $25^{\circ} \mathrm{C}$ and $50^{\circ} \mathrm{C}$. Data are presented as grams explosive per square millimeter. The values reported are repeatable within a factor of two.

TNT

\begin{tabular}{|c|c|c|c|c|}
\hline & \multicolumn{2}{|c|}{ 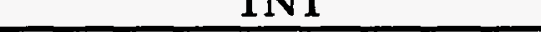 } & \\
\hline & $25^{\circ} \mathrm{C}$ & $50^{\circ} \mathrm{C}$ & $25^{\circ} \mathrm{C}$ & $50^{\circ} \mathrm{C}$ \\
\hline Substrate & & & & \\
\hline 6061-T6 Aluminum & $5.9 \times 10^{-13}$ & $1.62 \times 10^{-14}$ & $>1.06 \times 10^{-9}$ & $1.20 \times 10^{-12}$ \\
\hline Manila Envelope & $1.50 \times 10^{-13}$ & $1.62 \times 10^{-14}$ & $>1.06 \times 10^{-9}$ & $7.90 \times 10^{-12}$ \\
\hline Cardboard Box $*$ & $5.00 \times 10^{-13}$ & $3.10 \times 10^{-14}$ & $>1.06 \times 10^{-9}$ & $7.60 \times 10^{-11}$ \\
\hline Briefcase & $>1.06 \times 10^{-9} *$ & $4.90 \times 10^{-14}$ & $>1.35 \times 10^{-9}$ & $5.57 \times 10^{-11}$ \\
\hline PVC & $4.00 \times 10^{-12}$ & $1.80 \times 10^{-14}$ & $>1.06 \times 10^{-9}$ & $8.00 \times 10^{-11}$ \\
\hline Wood (pine) & $2.83 \times 10^{-13}$ & $3.47 \times 10^{-14}$ & $>1.62 \times 10^{-9}$ & $8.78 \times 10^{-12}$ \\
\hline
\end{tabular}

* No visible deposit could be seen on the surface.

- A white deposit of RDX was visible on the surface, but could not be detected using the conditions listed in this paper.

* One side of the cardboard box was the typical brown color, while the reverse side was white. The detection limits were found to be the same on both sides.

\section{Detection Limit of TNT}

As shown in Table 1, a detection limit of approximately $10^{-13}$ grams of TNT per square millimeter was obtained for manila, cardboard, aluminum, and pine at $25^{\circ} \mathrm{C}$. 
The detection limit on the PVC was approximately one order of magnitude greater. If the surface temperature of the substrate is increased to $50^{\circ} \mathrm{C}$, the detection limits are decreased by approximately one order of magnitude to $10^{-14}$ grams of TNT per square millimeter.

The surface of the Airway briefcase severely limits our ability to detect TNT. As shown in Table 1, no TNT could be detected on this surface at a concentration of $1 \times 10^{-9}$ grams per square millimeter. One explanation for this behavior is that TNT is soluble in the plasticizer, and the resulting concentration gradient could transport the TNT away from the surface. It was noted that after depositing the explosive solution on the briefcase and allowing the solvent to evaporate, the area where the solvent had evaporated had a very dull appearance. In addition, a ring around the periphery of the deposition area could be seen. This ring has been identified (Fourier Transform Infrared Spectroscopy) as plasticizer that has been extracted by, and carried along by the acetone. Other possible explanations for the lack of a response for TNT should be considered. For example, the plastic, or a species present in the plastic, could interact with the TNT and decompose or permanently bind it to the surface. The actual mechanism responsible for the poor detection limit of TNT on the Airway briefcase at $25^{\circ} \mathrm{C}$ has not been determined at this time. At a surface temperature of $50^{\circ} \mathrm{C}$, the detection limit of TNT on the Airway surface is comparable to the TNT detection limit on the other substrates.

\section{Detection Limit of RDX}

At $25^{\circ} \mathrm{C}$, RDX presents significant difficulties for vapor-phase detection. RDX was not detected on the surfaces even though a deposit of RDX could be seen with the naked eye. A considerable amount of RDX was obviously present, and several mechanisms can be proposed to explain the lack of a detectable signal.

Given the low vapor pressure of RDX (equilibrium concentration $=0.0060$ parts-perbillion at room temperature), very little RDX vapor is present in the region immediately above the deposit even under the best of conditions. It is conceivable that air currents in the room dispersed the RDX vapor, thereby decreasing the amount that could be collected. The influence of air currents on sample collection was evaluated by fabricating a small shield to protect the sample from extraneous air 
movement. All four sides were shielded, and only a minimal portion of the top was exposed, but still, RDX could not be detected. It therefore appears that dispersion of the RDX vapor due to air currents is not the primary cause for the lack of signal.

A second possibility for the lack of a signal is that the time required for the RDX to reach equilibrium is relatively long, and sufficient vapor phase RDX had not yet been generated when the samples were taken. To address the question of the rate of vapor equilibration, $1.25 \times 10^{-7}$ grams of RDX was deposited on cardboard, manila, and Airway plastic. (This quantity approximates the $1.0 \times 10^{-9} \mathrm{~g} \mathrm{RDX} / \mathrm{mm}^{2}$ that could not be detected at $25^{\circ} \mathrm{C}$ as shown in Table 1.) The solvent was allowed to evaporate, the samples placed into pretreated scintillation vials, and the vials were sealed. An additional sample was prepared by depositing the RDX solution directly into a treated vial rather than onto one of the substrates. After the vapor was allowed to equilibrate in the closed vial for approximately one hour at $25^{\circ} \mathrm{C}$, a headspace sample was collected. RDX could not be detected in any of the vials. A duplicate set of samples was prepared and allowed to equilibrate for ten days at $25^{\circ} \mathrm{C}$, after which they were sampled. Again, RDX could not be detected in the headspace volume. Heating the vials that contained the samples to $35^{\circ} \mathrm{C}$ for 25 hours also proved to be unproductive as RDX was not detected under these conditions. A $20 \mathrm{ml}$ scintillation vial containing 750 milligrams of bulk, powdered RDX was also sampled. Upon initially opening the vial, vapor phase sampling produced a response for RDX. If this vial was allowed to remain open for fifteen minutes before sampling, however, no RDX could be detected in the headspace volume even though gross amounts of RDX were present. It appears that at $25^{\circ} \mathrm{C}$ the vapor pressure of RDX is too low to allow an adequate sample to be collected using the methods employed here.

At a surface temperature of $50^{\circ} \mathrm{C}, \mathrm{RDX}$ can be detected on all surfaces with reasonable sensitivity. Note that the decrease in signal seen when TNT was deposited on the Airway briefcase at $50^{\circ} \mathrm{C}$ does not appear with RDX. The solubility of RDX in the plasticizer could influence this parameter, as could other surface phenomena described earlier. 


\section{Concentration Dependence}

The detection limit is slightly dependent on the concentration of the solution used to prepare the deposit. As stated previously, the detection limit was determined by preparing a solution of a given concentration and depositing decreasing volumes until either the detection limit was reached, or until a response could still be seen, but the volume of solution deposited was 0.02 milliliters or less. In the latter case, a larger volume of a more dilute solution was used to deposit an equivalent amount of explosive over the same geometrical area, and the deposition process would be continued. As an example, if 0.10 milliliters of $1 \times 10^{-9}$ grams TNT per milliliter was deposited on a surface and yielded a peak, I would proceed to deposit 1.0 milliliters of $1 \times 10-10$ grams TNT per milliliter solution to provide the same amount of explosive on the surface. Although an equivalent quantity of explosive was deposited, the larger volume of lower concentration solution often did not yield a detectable peak.

This phenomenon was examined by sampling a deposit prepared from a solution, and then using a more concentrated solution to deposit an equivalent amount of explosive on the surface. It was found that the peak obtained from the concentrated solution was approximately 30 times as large as that obtained from the dilute solution. Analysis has shown that the difference is not due to adsorption of the explosive onto the glassware, and does not appear to be a function of the amount of solute used to prepare the deposit. The reason for this apparent discrepancy has not been determined.

\section{Uniformity of Deposition}

Visual examination of the substrates showed that the explosive was not uniformly deposited. For example, in some samples, such as the RDX at $25^{\circ} \mathrm{C}$, the amount of explosive deposited was sufficient to be seen visually. This deposit was obviously not uniform, but rather, more material was located along the perimeter than in the center. It can be reasonably argued that deposits that were not visible were similarly distributed. To evaluate the effect of this dispersion characteristic on the analysis, samples of TNT and RDX were prepared both by making one large deposit, which we know is not uniform, and by making numerous smaller deposits over an 
equivalent area. Although each individual small deposit is not uniform, the overall effect is an approximation of a uniform deposit. When sampled, both deposits yield identical responses, indicating that the uniformity of the deposit is not a significant factor when determining the detection limit. Since we essentially collected "integrated" samples, these results were expected.

\section{Detection Limit as a Function of Time}

The photodecomposition of explosives is a well-documented phenomenon, and exposure to both laboratory lighting and sunlight has been noted to cause a loss of explosives [3]. However, many of the TNT and RDX deposits were sampled numerous times over the course of several hours. The responses obtained from these samples were constant, indicating that the quantity of explosive deposited on the surface was not significantly perturbed by either the sampling process or by exposure to laboratory lighting.

Several TNT samples were analyzed repeatedly over a period of ten days. During this period, the samples were allowed to sit in the open on a lab bench. The laboratory used does not have windows, so the incident radiation was supplied entirely by fluorescent lighting. Again, there was no significant difference in the responses observed, indicating that the deposit was not significantly perturbed by exposure to laboratory lighting over this time period. The quantity of TNT present on the surface is apparently large enough that the amount that decomposes is insufficient.

\section{Effect of Surface Topology}

The SEM photographs shown in Figures 1 through 6 demonstrate the large difference in the surface topology of the various substrates examined. However, the surface roughness of the substrate does not appear to influence the detection limit to a detectable degree. For example, the surface finish of aluminum is such that its true surface area is significantly less than the true surface area of the manila envelope. Nevertheless, the minimum detection limits for a given geometric surface area were found to be similar. As described later in this report, however, the surface topography does influence the transfer of explosives from a hand to that surface. 


\section{METHODS TO REDUCE THE DETECTION LIMIT}

\section{Solvent Addition}

It has been reported that lightly spraying a surface with acetone where an explosive had been deposited, and collecting a sample while the surface is still visibly wet, can decrease the detection limit [5]. We have attempted to reproduce this phenomenon by spraying both manila and aluminum surfaces with acetone after RDX and TNT had been deposited. The sample collection was begun immediately while the surface was still wet. Although the surface appeared dry prior to termination of the 90 second collection process, the sample remained visibly wet for approximately 60 seconds, and the odor of acetone could still be detected on the manila after the sample collection was complete. Two sets of samples were examined in this manner. In the first set, the deposits were a factor of two below the minimum detection limit. If the acetone (solvent) treatment decreased the detection limit, a signal should have been seen. Unfortunately, the acetone treatment did not enhance the response enough to obtain detectable signals. In the second test, the deposits were approximately ten times the minimum detection limit. An increase in the signal strength would indicate that the acetone treatment reduced the detection limit. Again, the treatment with acetone did not increase the signal obtained from these samples. It appears that acetone treatment does not reduce the detection limit for RDX or TNT on manila or aluminum as there was no enhancement of the signal after the treatment.

\section{Touch Sampling}

A simple way to improve the detection limit is to use touch rather than vapor sampling. After vapor samples had been collected at the detection limit, the various materials were sampled by touching the surface for approximately one second. Invariably, large signals were obtained. For example, $1.5 \times 10^{-13}$ grams TNT per square millimeter was the minimum amount that could be detected reliably on a manila envelope at $25^{\circ} \mathrm{C}$ using vapor phase sampling. When this same deposit was sampled by touching the deposit, the signal obtained was two to three orders of magnitude larger. $R D X$, which could not be detected using vapor sampling at $25^{\circ} \mathrm{C}$, 
saturated the IMS for approximately ten minutes after a touch sample. Touch sampling provided an average of at least two orders of magnitude better sensitivity than vapor sampling.

\section{TRANSFER OF EXPLOSIVES BY HUMAN TOUCH}

The work described thus far utilized "synthetic" contamination. A more realistic scenario would be an examination of surfaces that had been contaminated by someone who had been preparing a clandestine device. Quantifying the amount of explosive transferred to a surface by this person is not possible, but a qualitative examination of the transfer is feasible. All of the following tests were done at room temperature.

A military demolition block of TNT was handled with bare hands for approximately 15 seconds. The hands were then vigorously brushed and clapped together for about ten seconds to dislodge any loose particles of TNT. The surface to be tested was then handled, after which it was vapor sampled. TNT that had been transferred from the hand could be detected on the manila envelope, the cardboard box, and the wood surface. TNT could not be detected on the aluminum, plastic briefcase, or the PVC.

A small plastic bag of RDX powder was handled in the same manner as the TNT charge described above. It was assumed that some RDX powder would be present on the exterior of the bag, and would therefore be transferred during handling. After clapping and brushing the hands, and attempting to transfer the residual RDX to the various surfaces, a vapor sample was taken. No RDX could be detected above any of the surfaces. On the basis of the results described earlier, the inability to detect transferred RDX was expected.

After taking the vapor samples, the surfaces used for the above tests were sampled by touching with the quartz tube. Using this sampling method, TNT could be detected on all of the surfaces. The signals obtained were more intense on the rougher surfaces, i.e., the manila, cardboard, and wood, indicating that the surface texture may aid in the transfer and/or retention of the explosive from the hand to 
the substrate. RDX was detected on all of the surfaces by touch sampling as well, with a slightly more intense signal being obtained from the rough surfaces.

After performing the hand-to-substrate transfers and analyses described above, a modification was made to the procedure. In addition to clapping and brushing the hands, soap and water was used to clean the hands before handling the various substrates. After this more thorough cleansing of the hands, neither TNT nor RDX could be detected on any of the surfaces with either the vapor sampling or the touch sampling method. Even though transferred explosives could not be detected on the substrates, a direct touch sampling of the hands showed a slight response for both TNT and RDX. Vapor sampling of the hands did not yield a detectable signal.

\section{SUMMARY}

This work has determined:

- The minimum detection limits of TNT and RDX on various substrates.

- That the minimum detection limit is a function of the temperature of the surface rather than the surface topography.

- That the surface topography, or roughness, aids in transferring explosive particles from a person's hands to the substrate.

- That additives in plastics can influence the detection limit of TNT to a significant degree.

- That the detection of RDX at ambient temperatures is limited by the low vapor pressure of this compound.

- That the concentration of the explosive solution used to prepare the deposit influences the minimum detection limit.

- That the uniformity of the deposit does not influence the detection limit.

- That the signal obtained from a deposit appears to be constant with repeated sampling for at least ten days.

- That the detection limit is not decreased by spraying the surface with acetone.

- That touch sampling provides two to three orders of magnitude better sensitivity than vapor phase sampling. 


\section{REFERENCES}

1. B. T. Kenna and F. Conrad, "Studies of the Adsorption - Desorption Behavior of Explosive Molecules, Internal Document, SAND86-0141

2. D. Ingersoll, P.J. Rodacy "Control of the Reactant Ion Chemistry for the Analysis of Explosives by Ion Mobility Spectroscopy," SAND Report in progress

3. P.J. Rodacy and D. Ingersoll, "The Collection, Handling, Transportation, and Thermal Desorption of Explosive Vapor Using Quartz Collection Tubes," SAND 90-1326

4. Adsorption of Explosives onto glass surfaces -- SAND Report in progress

5. Dr. F. W. Whitehurst, Federal Bureau of Investigation, Laboratory Division, Washington, D.C. Private Communication. 

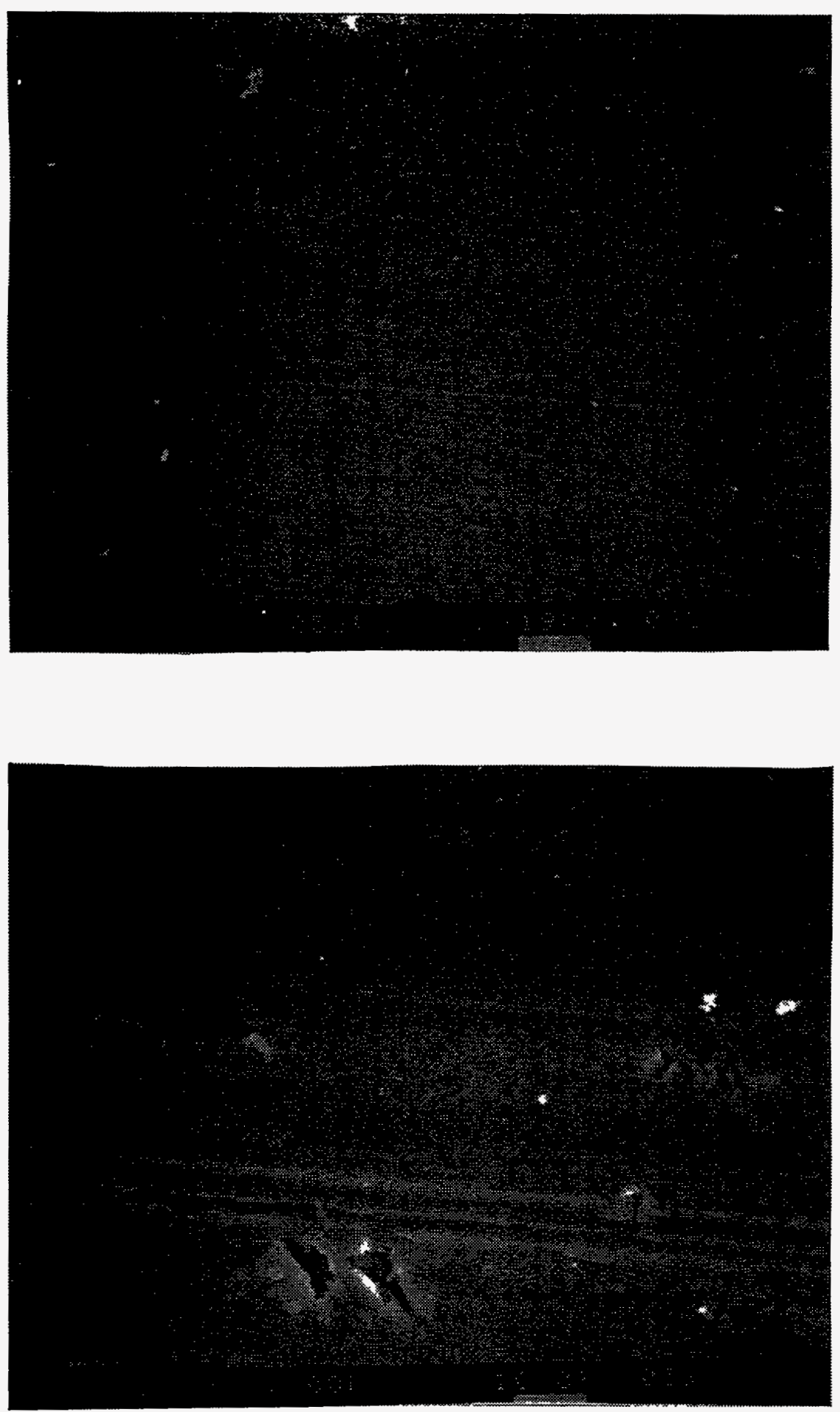

Figure 1. 6061-T6 Aluminum 

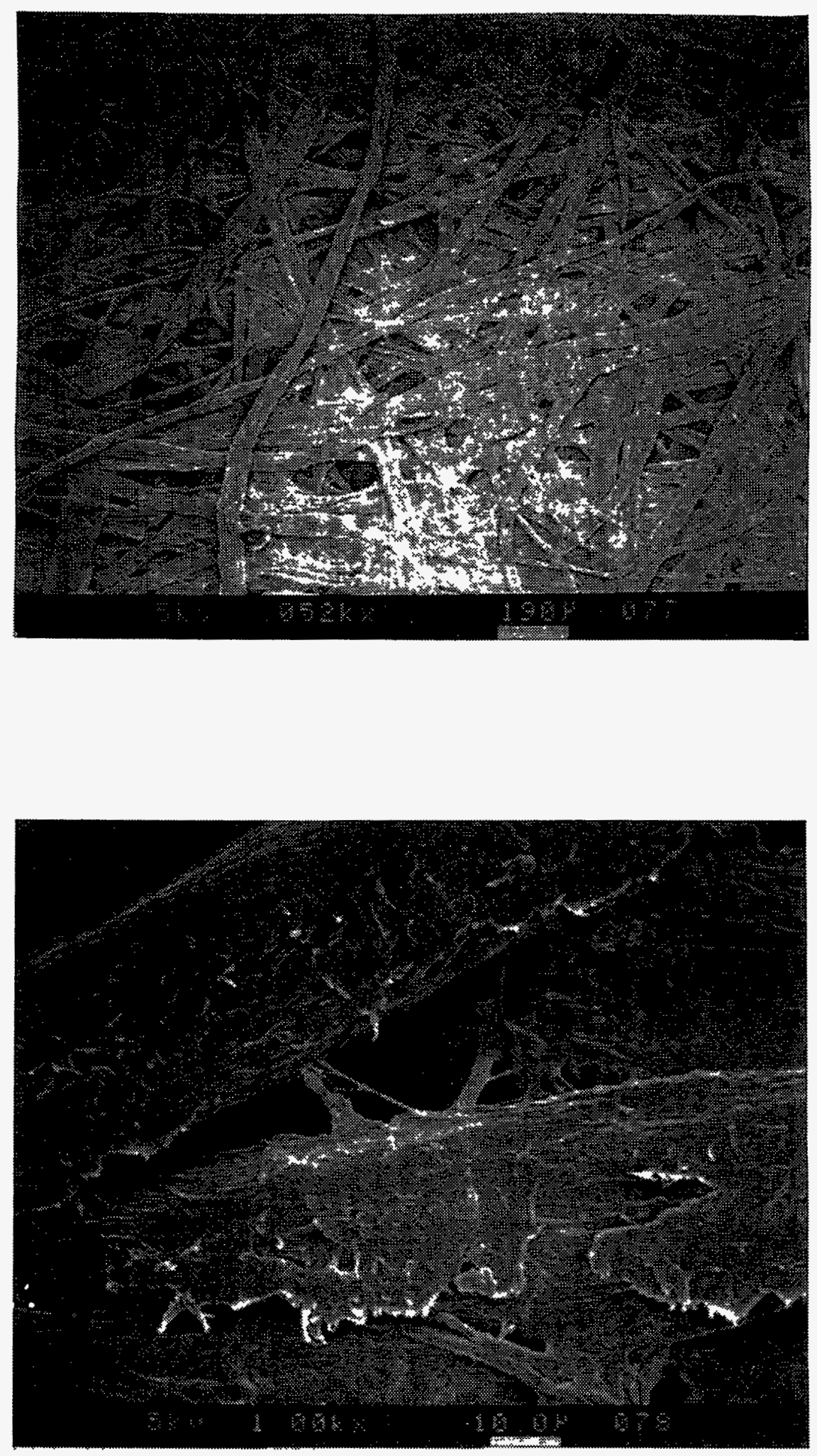

Figure 2. Manila Envelope 

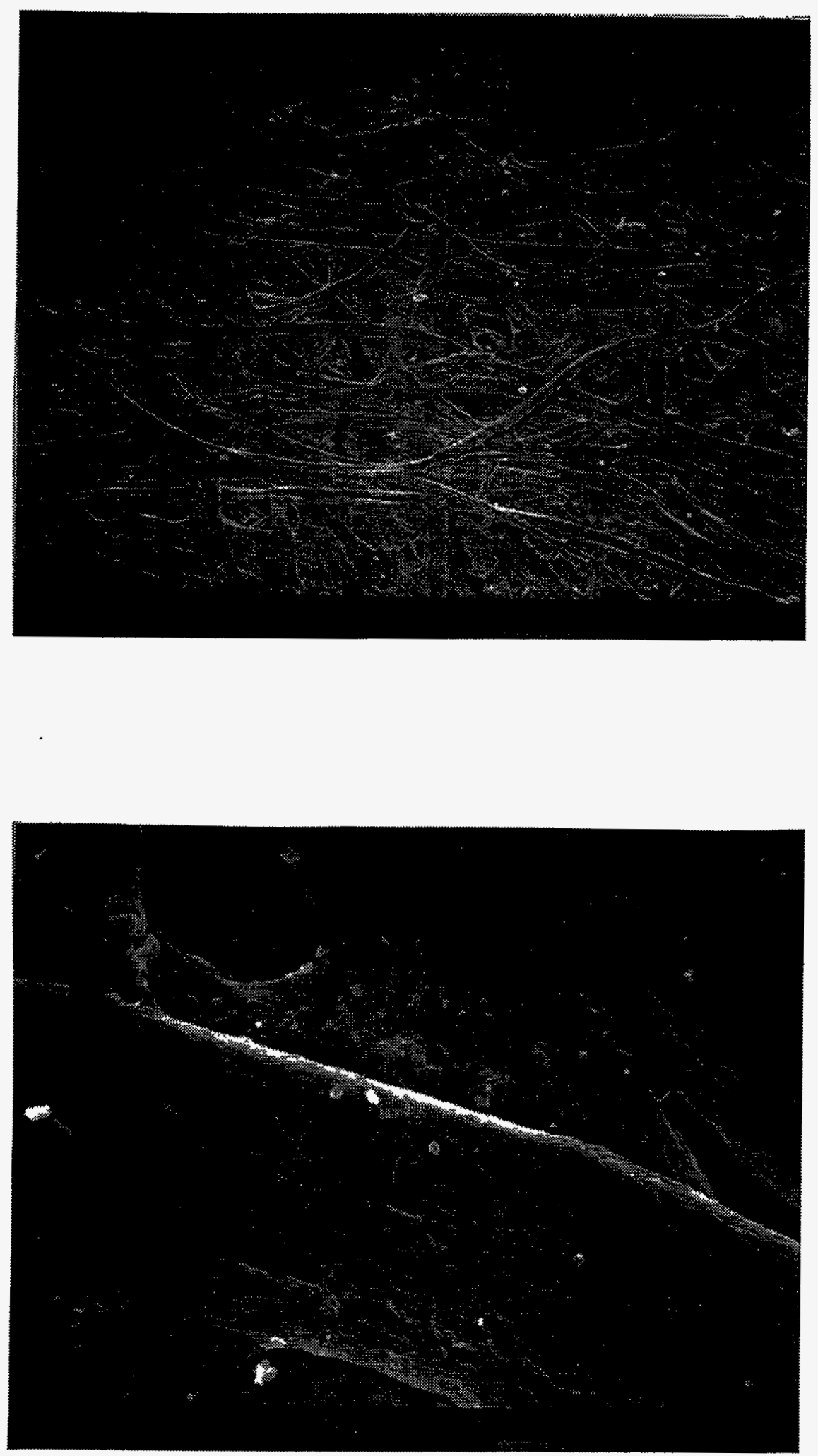

Figure 3. Cardboard Box 

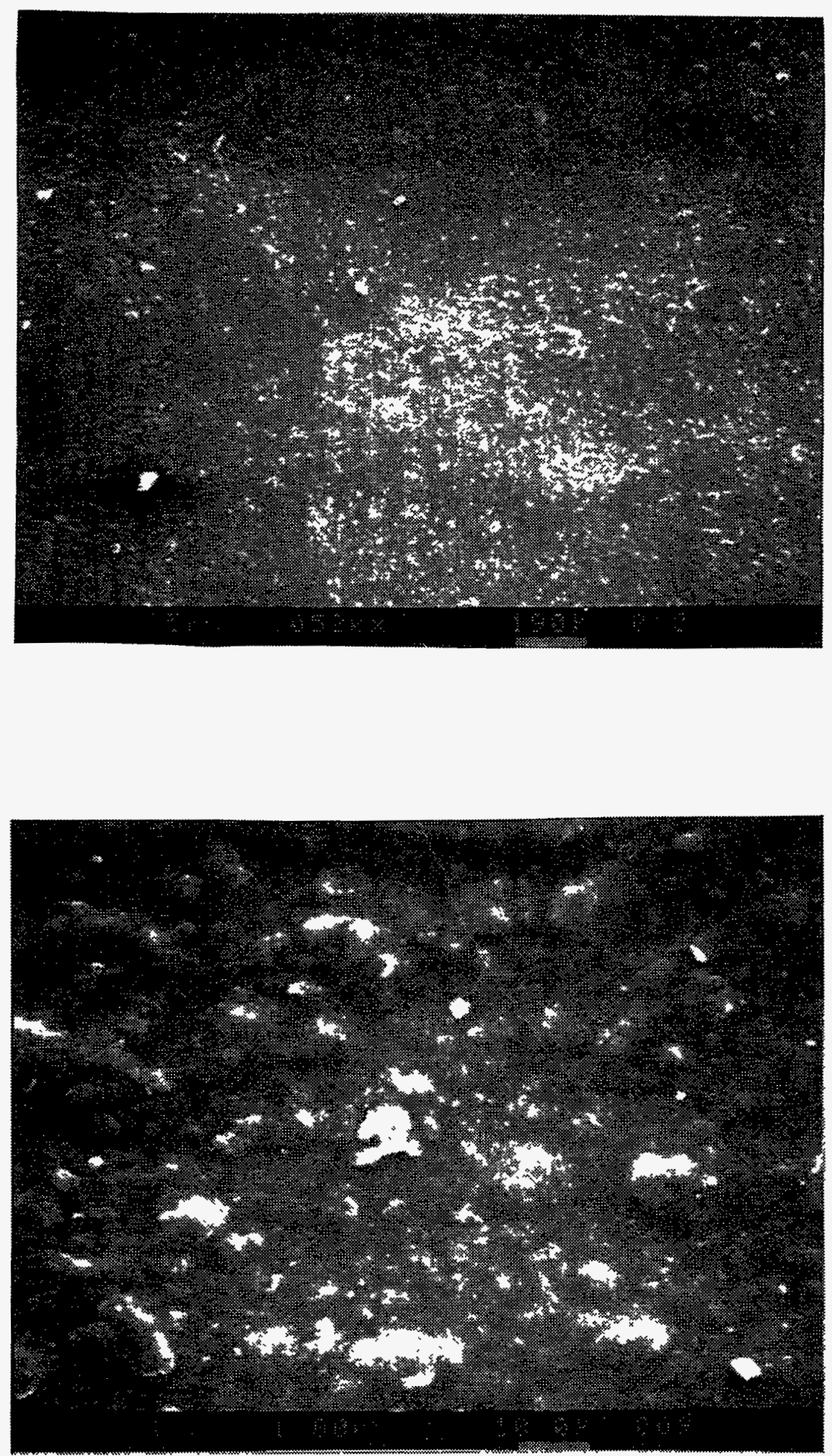

Figure 4. Airway ${ }^{\circledR}$ Briefcase 

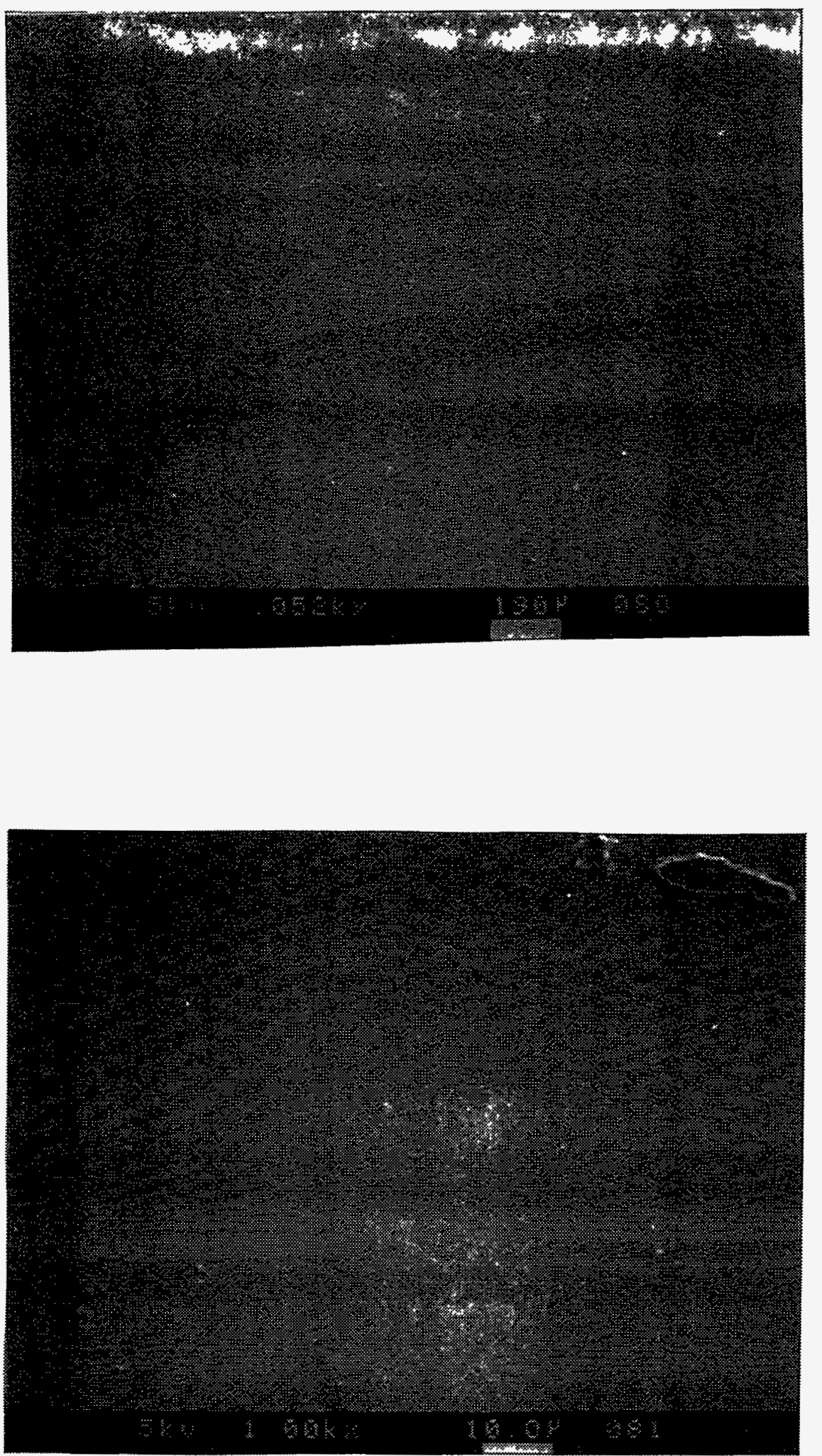

Figure 5. Poly(vinylchloride) (PVC) 

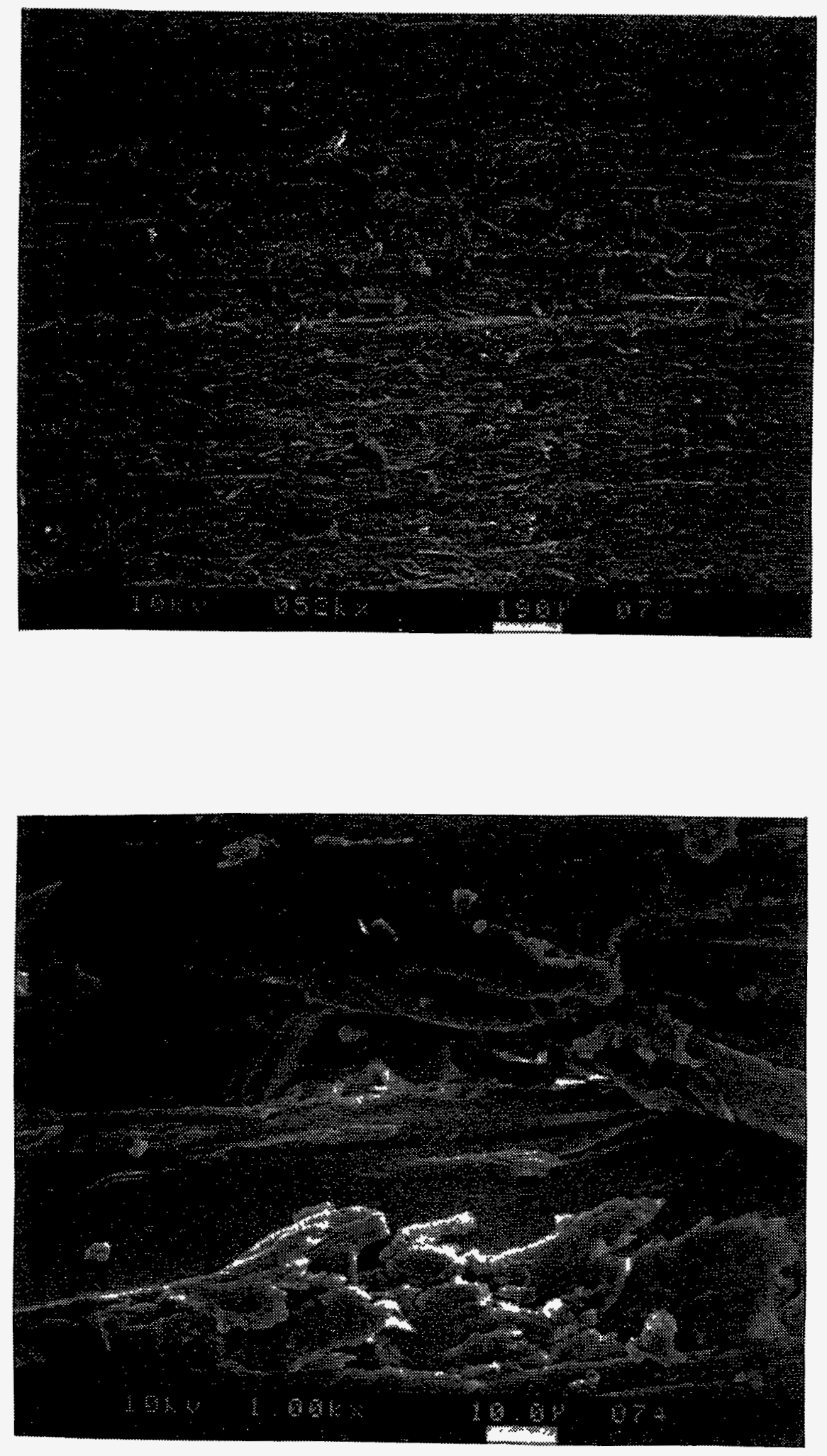

Figure 6. Pine 


\section{DISTRIBUTION}

Dr. Dean Fetterolf

FBI Academy

Forensic Science Research \&

Tining Center

Quantico, VA 22135

Dr. Gary Glish

Oak Ridge National Laboratory

Y-12 Plant

Oak Ridge, TN

Dr. John Hobbs

Transportation Systems Ctr.

Department of Transportation

Kendall Square

DTS-75

Cambridge, MA 02142

Dr. Scott McLuckey

Oak Ridge National Laboratory

Y-12 Plant

Oak Ridge, TN

Dr. Frederic W. Whitehurst

Federal Bureau of Investigation

Laboratory Division

JEH Building

10th \& Pennsylvania, N.W.

Washington, D.C. 20535
2512 J. G. Harlan

102512 P. J. Rodacy

9548 J. F. Chapek

59548 F. J. Conrad

9548 D. W. Hannum

9548 B. T. Kenna

8523-2 Central Technical Files

57141 Technical Library

10 7613-2 Document Processing for DOE/OSTI

7151 Technical Publications 